\title{
HIVFactSheet: A mobile application designed and implemented by youth peer mentors to facilitate HIV and reproductive health care among adolescents and young adults
}

\section{Edith Apondi}

Moi Teaching and Referral Hospital

Salim Bakari

AMPATH Kenya

Brian Kwendo

AMPATH Kenya

Jones Ingari

University of Nairobi

Carole McAteer

AMPATH Kenya

Michael Scanlon

Icahn School of Medicine at Mount Sinai

Josephine Aluoch

AMPATH Kenya

Judith Toromo

AMPATH Kenya

Jane Chemon

AMPATH Kenya

Sylvester Kimaiyo

AMPATH Kenya

Winstone Nyandiko

Moi University College of Health Sciences

Rachel C. Vreeman

Icahn School of Medicine at Mount Sinai

Leslie A. Enane ( $\boldsymbol{D}$ lenane@iu.edu )

Indiana University School of Medicine https://orcid.org/0000-0001-8390-5881 
Keywords: adolescents, mHealth, mobile applications, HIV/AIDS, sexually transmitted infections, reproductive health, pregnancy, health education, sub-Saharan Africa

Posted Date: March 3rd, 2020

DOI: https://doi.org/10.21203/rs.3.rs-15674/v1

License: (c) (1) This work is licensed under a Creative Commons Attribution 4.0 International License. Read Full License 


\section{Abstract}

Background:Adolescents and young adults in sub-Saharan Africa face complex challenges in accessing HIV testing, HIV care, and sexual and reproductive health services. We describe and characterize the uptake of a unique mHealth intervention that was designed, led, and implemented by youth peer mentors (YPM) in the Academic Model Providing Access to Healthcare (AMPATH) in western Kenya.

Methods: YPM developed a mobile application to address the unmet needs that they identified in youth education around HIV testing, HIV care, and sexual and reproductive health. The application incorporates comprehensive educational materials and information about health services in Kenya. Users can set up reminders for clinic appointments and may call, text, or email a YPM for assistance accessing care or to ask care-related questions. The application has been promoted at AMPATH clinics and on social media. We describe its implementation in this setting.

Results: Since launching the application in December 2017, as of April 2019 the application was downloaded 5,800 times, with 4,953 current users. Downloads primarily originated in Kenya (74\%) and other African countries, but also globally. Texts to YPM through the application included questions about a broad range of topics on HIV testing, prevention, and care; reproductive and sexual health; substance abuse and addiction; and navigating health care. YPM contacted through the app provided individualized counseling and referred 183 users to health services. YPM utilize the application in large- and small-group and individual peer education sessions, and in trainings for healthcare workers, teachers and new YPM.

Conclusions: Adolescents and young adults should be empowered to lead interventions to address the challenges that they face in navigating care. A unique mobile application intervention spearheaded by YPM providing access to comprehensive health education and individualized counseling and referral has great potential to facilitate HIV and sexual and reproductive health care for young people.

\section{Background}

The current generation of adolescents and young adults (AYA, ages 10 to 24), is the largest in history, at 1.8 billion, and is growing fastest in low- and middle-income countries, where $90 \%$ of AYA live. $(1,2)$ Fostering AYA health has great importance to the well-being of AYA, to the health of future generations, and to economic development, yet has been a neglected area.(3) HIV/AIDS and maternal disorders are leading causes of death among AYA, and unsafe sex and drug and alcohol use are major risk factors for reduced disability-adjusted life-years. $(3,4)$ AYA have low rates of HIV testing, and those living with HIV infection have complex challenges related to their engagement and retention in care and adherence to antiretroviral therapy.(5-8) Consequently, AYA face poor HIV outcomes and have been the only age group with increasing HIV-associated mortality in recent years.(9-11) AYA may also experience limited access to sexual and reproductive health $(\mathrm{SRH})$ care and education, particularly access to and uptake of contraceptive methods. $(12,13)$ Meanwhile, adolescent pregnancies and maternal disorders including complications of unsafe abortion are unacceptably prevalent.(14) 
Kenya has had extremely high uptake of mobile phones and a history of innovation in mobile technology. Leading the continent in smartphone penetration, over $90 \%$ of Kenyans have a mobile phone subscription, and mobile data subscriptions account for $99 \%$ of the 30.8 million internet subscriptions. $(15,16)$ Mobile phones, particularly smart phones, offer an alternative media format for targeting AYA (both in and out of school) for high quality education and messaging about HIV and SRH. In addition, the interactive nature of this technology offers the opportunity for AYA to reach out for further information, and to feel supported.(17) AYA may be more likely to ask difficult questions through a mobile or tablet interface, rather than in-person to a health professional.(15) This may be particularly true for AYA in Kenya and other countries of sub-Saharan Africa, where AYA may be highly deferential to health professionals and hesitant to raise questions or concerns related to SRH. $(18,19)$ Further, mHealth interventions potentially provide an avenue to maintain communication with AYA, who represent a highly mobile population who are at higher risk of loss to follow-up and disengagement from medical care, and to facilitate care and access for this group.

There has been considerable interest in mHealth interventions to improve adolescent engagement in HIV and SRH care.(20-24) Much of this literature originated from the United States and other high-income countries.(20) Here we describe and characterize the uptake of a unique mHealth intervention incorporating both HIV and SRH education with a platform for accessing youth peer mentor (YPM) support and care referral, that was designed, led, and implemented by Kenyan YPM.

\section{Implementation}

\section{Setting}

YPM are young adults who are engaged in peer mentorship to AYA navigating HIV and SRH care. YPM in the Academic Model Providing Access to Healthcare (AMPATH) program receive dedicated training in HIV education and adolescent health, and liaise with a multidisciplinary care team at AMPATH clinics to facilitate adolescent care. AMPATH provides HIV care to approximately 160,000 people living with HIV throughout western Kenya.(25) The HIVFactSheet mobile application was independently developed by YPM in AMPATH who are associated with the Rafiki Center for Excellence Adolescent Health at Moi Teaching and Referral Hospital (MTRH), one of the leading institutional partners of AMPATH, in Eldoret, Kenya. The Rafiki Center provides comprehensive adolescent health services, including HIV testing and treatment, provision of pre-exposure prophylaxis (PrEP), and SRH care.

\section{Mobile Application}

YPM identified a need to develop a mobile application (app) to provide educational resources on HIV and $\mathrm{SRH}$, as well as access to AYA at AMPATH to assist in navigating care. They consulted with an HIV and SRH technical advisory team at AMPATH and MTRH, which included three pediatric physicians specializing in HIV care, a clinical officer, and five YPM. The advisory team reviewed and approved app content for both accuracy and appropriateness for AYA populations and also served as a consultation service to answer clinical and other questions that arose through texts, calls, and emails by clients using 
the app. YPM in AMPATH also reviewed the content and gave input on needed resources in the app through individual and group discussions. Educational content for the app was provided by Kenyan state bodies, including the Kenyan Ministry of Health, the National AIDS and STI Control Program, and National AIDS Control Council; as well as several NGOs including International Youth Alliance for Family Planning, LVCT Health, and Q Initiative. In addition, short films and narratives related to HIV disclosure and stigma (available at www.hiv-films.org) developed as part an $\mathrm{NIH}$-funded study were also available through the app.(26)

The HIVFactSheet app is available for free download on Android mobile devices through the Google Play store (Fig. 1). Once downloaded, the app operates offline, which was important in this setting as many AYA cannot afford to use apps that require significant amounts of mobile data. The app is also now available on an online platform (available at https://hivfactsheet.org). Information in the app is categorized in several content sections (Table 1).

Table 1

Content sections and sub-topics currently within the HIVFactSheet mobile application developed by youth peer mentors in western Kenya.

\begin{tabular}{|ll|}
\hline Content Sections & Sub-Topics within Each Section \\
\hline Facts about HIV & $\begin{array}{l}\text { HIV overview } \\
\text { Healthy living } \\
\text { HIV testing and treatment } \\
\text { HIV transmission and prevention } \\
\text { Disclosure } \\
\text { HIV and TB } \\
\text { HIV and family }\end{array}$ \\
\hline Reproductive Health & $\begin{array}{l}\text { Anatomy and physiology } \\
\text { Sex and sexuality } \\
\text { Sexual / gender-based violence } \\
\text { Family planning and contraceptives } \\
\text { Menstruation and conception }\end{array}$ \\
\hline Teenage pregnancy \\
Gender and gender identity
\end{tabular}

With the app, users can set up reminders for clinic appointments. The app also provides a national-level referral directory with contact information for public and private health facilities, mental health centers, 
rehabilitation centers, and care centers providing HIV and SRH care throughout Kenya. A portal in the app allows users to call, text, or email a YPM directly for individualized counseling and referral. All communications through the app were fielded by the YPM app developers (SB and BK), who provided individualized counseling and answered specific health care questions. When YPM developers did not know or were not confident in an answer, they consulted with the technical advisory team through a regular WhatsApp chatroom where advisors were regularly available to respond to urgent questions and referral requests.

To increase awareness of the app among AYA at AMPATH, YPM began informing AYA about the HIVFactSheet app as part of individual peer support sessions as well as at social 'fun days' at Rafiki Center (Fig. 2). After an initial 3-month period of exclusively promoting the app at Rafiki Center, the potential user base was expanded through promotion of the app on multiple social media sites including Facebook and Twitter. YPM then presented the app in fun days for AYA at several surrounding AMPATH sites (including MTRH, Kisumu, Chulaimbo, Busia, Kitale, among others).

\section{Analysis}

We describe the uptake and utilization of the HIVFactSheet app through summary routine data provided by Google to app developers. We quantified the number of app downloads and active users; communications through the app via calls, texts, and emails; the number of individual referrals made to health facilities; and activity on our social media pages. We also reviewed a deidentified set of texts that had been sent to YPM counselors through the app portal and categorized the types of questions and resources sought by users. Finally, we describe the uptake of the app within YPM and staff training, and routine YPM counseling services in the AMPATH program.

\section{Ethics}

The HIVFactSheet app was independently developed and implemented by YPM and not conceived of as part of a research study. Users are not identifiable through the app unless they use the app to call or text a counselor. Individual names, phone numbers, emails, or any other identifiable information were not recorded. Google provides the app developers with limited user data regarding number of app downloads and deletions, and country of download. We consulted the local Kenyan institutional review board (IRB), the Moi University / Moi Teaching and Referral Institution Research and Ethics Committee, in Eldoret, Kenya, and the Indiana University School of Medicine IRB, in Indianapolis, Indiana, USA, where the senior author (LAE) is employed, who advised that IRB approval was not required for description of the app and its uptake in routine YPM counseling services in the AMPATH program.

\section{Results}

The HIVFactSheet app was launched in December 2017 and as of April 2019 it had been downloaded 5,800 times, with 4,953 current users. It is estimated that during the first three months, downloads were limited to AYA in care at Rafiki Center as this is where we first publicized the app, and there were 832 downloads during this initial time period before wider promotion of the app. Overall, user downloads 
primarily originated from Kenya (74\%), but also from Nigeria (3\%), Tanzania (3\%), Uganda (3\%), South Africa (3\%), and other countries. Through the app the YPM team received 101 calls, 197 texts, and 41 emails (Table 2).

Table 2

Communications and interactions with youth peer mentors through the HIVFactSheet app during the time period from December 1, 2017 to April 30, 2019, by mode of communication.

\begin{tabular}{|ll|}
\hline Mode of communication & Communications, $\mathbf{n}(\%)$ \\
\hline Telephone calls & $101(29.8 \%)$ \\
\hline Emails & $41(12.1 \%)$ \\
\hline Text & $197(58.1 \%)$ \\
\hline TOTAL & 339 \\
\hline
\end{tabular}

The content of messages sent to YPM included questions regarding a broad range of health topics (Table 3). HIV-related themes included questions regarding HIV transmission, prevention (including PrEP), and symptoms; opportunistic infection (OI) prevention; antiretroviral treatment; HIV disclosure and stigma; pregnancy and HIV; and healthy living with HIV. SRH-related questions were similarly wideranging, including menstruation, sexually transmitted infections (STI), sexuality, healthy relationships, pregnancy, family planning, and abortion. Other topics included dealing with addiction to drugs and alcohol (in the context of HIV infection or otherwise), communication with parents or with partners, and accessing health information and services for issues that were difficult to discuss with health providers. YPM provided counseling and referral as appropriate, with support from clinical providers as needed. YPM referred 183 individuals (97 males and 86 females) to health facilities for evaluation and care. 
Table 3

Example content areas of questions sent to youth peer mentors through the HIVFactSheet app.

$\begin{array}{lll}\text { Topic Sub-Topic } & \text { Example Content Areas and Questions } \\ & \text { Sent to Youth Peer Mentors }\end{array}$

HIV

transmission

HIV prevention

HIV treatment

PrEP

Prevention with positives

Risk of HIV transmission

ARVs

Lifespan

Symptoms

Disclosure to friends or others

Disclosure to sexual partner

HIV disclosure

Nutrition

Positive living

Substance use and HIV

Relationships and sex

Resilience

Self-stigma

External stigma

Substance

abuse

STIS
Dealing with addiction

Symptoms
- How to stop losing weight

- How to protect negative sexual partners of individuals living with HIV infection

-What to do after unprotected sex between positive and negative sexual partners

- Viral load and risk of HIV transmission

- Contact with body fluids

- Unprotected sex

- Where to get PrEP

- Do ARVs have adverse effects on menses, pimples, sexual desire or erectile dysfunction?

- If a dose of ARVs is missed, should that day be skipped?

- Are injectable ARVs available?

-What to do if dapsone dose is missed

- Role of cotrimoxazole prophylaxis

-What is the lifespan of child born with HIV?

- Frequent mouth sores, vomiting, decreased appetite

- How to navigate disclosure to friends

- Disclosure to school staff

- Disclosure after unprotected sex

- How to overcome alcohol addiction

- How to stop smoking tobacco

- Disclosure, sexuality, having a family, navigating relationships with HIV-negative persons

- Coping with broken relationships

- Dealing with self-stigma related to school

- Dealing with external stigma in school

- How to overcome alcohol addiction

- How to stop smoking tobacco

- Urethral discharge

- Lower abdominal pain

Abbreviations: ARVs, antiretroviral medications; Ol, opportunistic infection; PrEP, pre-exposure prophylaxis; STIs, sexually-transmitted infections. 


\begin{tabular}{|c|c|c|}
\hline Topic & Sub-Topic & $\begin{array}{l}\text { Example Content Areas and Questions } \\
\text { Sent to Youth Peer Mentors }\end{array}$ \\
\hline & Treatment & - How are STIs treated \\
\hline \multirow[t]{2}{*}{$\begin{array}{l}\text { Family } \\
\text { planning }\end{array}$} & $\begin{array}{l}\text { Emergency } \\
\text { contraception }\end{array}$ & -What to do after unprotected sex \\
\hline & $\begin{array}{l}\text { Contraception and } \\
\text { family planning }\end{array}$ & $\begin{array}{l}\text { - Where can adolescents access family planning methods } \\
\text { - What is the risk of pregnancy using the withdrawal } \\
\text { method }\end{array}$ \\
\hline \multirow[t]{2}{*}{ Pregnancy } & $\begin{array}{l}\text { Diagnosis of } \\
\text { pregnancy }\end{array}$ & $\begin{array}{l}\text { - What to do if missed menses } \\
\text { - How did pregnancy happen? What to do next? } \\
\text { - What to do if the pregnancy is not wanted }\end{array}$ \\
\hline & HIV and pregnancy & $\begin{array}{l}\text { - What to do if positive and wanting to have children } \\
\text { - What to do if in a sero-discordant relationship and } \\
\text { wanting to have children } \\
\text { - How HIV affects pregnancy }\end{array}$ \\
\hline Abortion & & - How to obtain an abortion \\
\hline \multirow{4}{*}{$\begin{array}{l}\text { Menstrual } \\
\text { period }\end{array}$} & Normal menses & - Cycle and duration of menses \\
\hline & Dysmenorrhea & - Is dysmenorrhea before menses normal? \\
\hline & $\begin{array}{l}\text { Menses and HIV } \\
\text { transmission }\end{array}$ & -What is the risk of HIV transmission during menses? \\
\hline & Menses and ARVs & - Are menses affected by ARVs? \\
\hline Relationships & $\begin{array}{l}\text { Navigating } \\
\text { relationships and } \\
\text { break-ups }\end{array}$ & $\begin{array}{l}\text { - Can a HIV-positive person marry a HIV-negative person? } \\
\text { - What to do if partner is "cheating" } \\
\text { - What to do if not interested in a relationship } \\
\text { - How to break off a relationship } \\
\text { - What to do after a broken relationship }\end{array}$ \\
\hline $\begin{array}{l}\text { Sex and } \\
\text { sexuality }\end{array}$ & Sexual desire & $\begin{array}{l}\text { - Do ARVs increase sexual desire? } \\
\text { - Do ARVs cause erectile dysfunction? } \\
\text { - Can a broken relationship cause erectile dysfunction? }\end{array}$ \\
\hline $\begin{array}{l}\text { Communication } \\
\text { with parents }\end{array}$ & Difficult topics & $\begin{array}{l}\text { - Difficulties talking with parents about relationships, } \\
\text { sexuality, STIs, erectile dysfunction, broken relationships }\end{array}$ \\
\hline $\begin{array}{l}\text { Accessing } \\
\text { medical care }\end{array}$ & Difficult topics & $\begin{array}{l}\text { - Difficulties discussing needs with providers, regarding } \\
\text { e.g. STIs, family planning needs, erectile dysfunction }\end{array}$ \\
\hline $\begin{array}{l}\text { Abbreviati } \\
\text { prophylaxi }\end{array}$ & al me & $\begin{array}{l}\text { ons; OI, opportunistic infection; PrEP, pre-exposure } \\
\text { ctions. }\end{array}$ \\
\hline
\end{tabular}

The AMPATH care program incorporated the HIVFactSheet app into the training of new YPM who are positioned to liaise with AYA and multidisciplinary teams at multiple AMPATH clinics across western Kenya. YPM have been trained on using the educational material in the app in their work to support AYA in navigating HIV and SRH care. YPM present the app in formal trainings for new YPM and adolescent 
healthcare workers throughout the AMPATH program in western Kenya. In presentations to groups of parents or caregivers, YPM present content on the app as part of training and support for disclosure to perinatally-infected children and adolescents. All training sessions include demonstration of HIV stigma and disclosure video content, to promote awareness and discussion around these issues. In Trans Nzoia County, YPM have presented the app in presentations to community groups of schoolteachers, community health volunteers, and religious leaders who regularly work with AYA.

Teaching around app educational content has been formalized in YPM-led peer group sessions with AYA, in which participants are assigned to lead a teaching session and discussion around dedicated topics, referencing materials provided in the app. In one-on-one counseling sessions with AYA, YPM inform AYA of the content provided by the app, as well as its utility for accessing YPM for further support.

The YPM developers and clinical consultants meet on a regular basis to discuss progress in implementing the app and areas where updates are desired, which are implemented by the developers on an iterative basis. Changes have been made to update content and to make the language more approachable to AYA. Further work may be needed to revise content to further resonate with AYA in African settings.

\section{Discussion}

The HIVFactSheet app represents a potentially powerful YPM-led intervention targeting AYA that provides a platform for comprehensive education on HIV and SRH care and services, functions such as reminders for clinic visits, and access to individualized counseling and referral by trained YPM. Here we describe the design and implementation of this app as part of routine YPM counseling services to AYA at a large HIV care program, AMPATH, in western Kenya. This mHealth intervention demonstrates the power of YPM to develop integral solutions to address gaps in care for AYA.

While not all AYA have access to a smart phone, in our setting in Kenyan YPM were able to use the app to support education and counseling services that they provide to AYA as part of a multidisciplinary care approach. The app demonstrates the potential for using mobile applications to support the work of YPM and other HIV educators in resource-limited settings. This type of intervention may be of particular utility for clinical programs looking to scale up AYA peer support programs, including to clinical sites where dedicated adolescent health-trained clinicians and professionals may not be available. Use of such an app by YPM may facilitate scale-up of YPM counseling through expanded access to educational information and referral resources when used by YPM in clinical sites distant from more comprehensive adolescent health care. Use of this type of application may include use by school teachers or counselors, or others engaged in HIV and SRH education among AYA. Teachers have reported a lack of appropriate content and training as a barrier to delivery of SRH education. $(27,28)$ Mobile applications like the HIVFactSheet represent a readily scalable platform to disseminate high quality HIV and SRH education, and to access highly trained and experienced YPM for further information where needed. 
There is a critical need to identify innovative strategies to expand HIV and SRH education and service uptake among AYA.(29) In Kenya, $15 \%$ of females and $22 \%$ of males have their sexual debut before age 15, and reported condom use is low.(30) AYA may have misconceptions about contraception,(13) and community support for AYA access to SRH may be lacking in some contexts.(12) Strategies are needed to provide direct, high-quality HIV and SRH education to AYA in a youth-friendly format that meets their needs in terms of the mode of communication, language appropriateness, cost, and privacy. We present a YPM-led mobile application designed to meet AYA needs and support both high-quality SRH education and service uptake.

Mobile platforms can provide autonomy for the user to seek and access information as needed, as well as communication with peers and health providers. A mobile platform targeted at improving HIV and SRH knowledge among AYA in sub-Saharan Africa could provide much-needed education, even to those not currently accessing HIV services, particularly in countries like Kenya were mobile phone use is very high. This provides a means of reaching AYA who may be in or out of school, who are seeking high-quality education about HIV and SRH.

Several studies have evaluated a range of mHealth interventions and their effect on HIV testing, ART adherence, and HIV prevention.(31-35) While what we present here is not a research study and we are not able to report on the effect of app use on AYA care outcomes, such as HIV and STI testing, ART adherence, and uptake of HIV and SRH services, we describe the incorporation of the HIVFactSheet app to enhance the important work of YPM to support AYA accessing and navigating care. The impact of such an intervention may be evaluated in future research studies, including studies in which smart phones may be provided to access the app. We suggest that such an intervention can support dissemination of highquality HIV and SRH education, including information about HIV testing and access to PrEP, and individualized counseling and referral to a range of "hard-to-reach" AYA. These may include AYA who are street-connected or living in informal settlements and AYA members of key populations. We highlight the potential of this type of app to be used as part of provision of AYA peer support, as it is being rolled out for this purpose in the AMPATH care program.

\section{Conclusions}

AYA should be empowered to lead interventions to address the challenges that they face in navigating care. Increasing HIV and SRH education to AYA and supporting them to access testing and care is an urgent priority, and innovative solutions are needed to address AYA challenges in accessing care. The HIVFactSheet app intervention was spearheaded by YPM and provides access to comprehensive health education and individualized counseling and referral, and has great potential to facilitate HIV and SRH care for young people.

\section{List Of Abbreviations}

AMPATH Academic Model Providing Access to Healthcare 
AYA adolescents and young adults

MTRH Moi Teaching and Referral Hospital

Ol opportunistic infection

PrEP pre-exposure prophylaxis

SRH sexual and reproductive health

STI sexually transmitted infections

YPM youth peer mentors

\section{Declarations}

\section{Availability and Requirements}

Project name: HIVFactSheet

Project home page: https://hivfactsheet.org

Operating system(s): Android 4.0 and up; online website platform

Ethics approval and consent to participate: Ethics approval was waived as this project did not meet criteria for human subjects research.

Consent for publication: Not applicable.

Availability of data and materials: Not applicable.

Competing interests: The authors declare that they have no competing interests.

Funding: Dr. Enane is supported by the Eunice Kennedy Shriver National Institute of Child Health \& Human Development of the National Institutes of Health under Award Number K23HD095778. This work was additionally supported by the Thrasher Research Fund, the Indiana Center for AIDS Research, and the Indiana Clinical and Translational Sciences Institute, funded, in part by Award Number UL1TR001108 from the National Institutes of Health, National Center for Advancing Translational Sciences, Clinical and Translational Sciences Award. HIV stigma and disclosure videos and content for the mobile application were developed under National Institutes of Health National Institute of Mental Health Award Number R01MH099747. The content of this manuscript is solely the responsibility of the authors and does not necessarily represent the official views of the National Institutes of Health.

Authors' contributions: SB and BK developed the mobile application. EA, JC, WN, and LAE serve on a clinical consultation group of clinicians supporting the app. SB, BK, EA, JI, CM, MS, JA, JT, JC, SK, WN, 
RCV, and LAE contributed to app content and/or implementation of the app within the clinical care program. EA and LAE drafted the manuscript. All authors contributed to the preparation of the final manuscript.

Acknowledgments: We acknowledge the remarkable contributions of young people to the fight against HIV and for sexual and reproductive health care. We acknowledge and thank the clinicians, youth peer mentors, and local and national organizations that provided content for the HIVFactSheet mobile application, including the Kenyan Ministry of Health, National AIDS and STI Control Program, and National AIDS Control Council; the International Youth Alliance for Family Planning, LVCT Health, and Q Initiative. We also acknowledge all those who participated in the promotion and dissemination of the mobile application.

\section{References}

1. Sawyer SM, Afifi RA, Bearinger LH, Blakemore S-J, Dick B, Ezeh AC, et al. Adolescence: a foundation for future health. The Lancet. 2012;379(9826):1630-40.

2. UNFPA. The Power of 1.8 Billion. UNFPA State of World Population 2014; 2014 Oct 31.

3. Mokdad AH, Forouzanfar MH, Daoud F, Mokdad AA, El Bcheraoui C, Moradi-Lakeh M, et al. Global burden of diseases, injuries, and risk factors for young people\&apos;s health during 1990-2013: a systematic analysis for the Global Burden of Disease Study 2013. The Lancet. 2016;387(10036):2383-401.

4. WHO. Health for the World's Adolescents: A second chance in the second decade Geneva, Switzerland: World Health Organization; 2014 [updated May 10. Available from: http://www.who.int/maternal_child_adolescent/documents/second-decade/en/.

5. Enane LA, Davies M-A, Leroy V, Edmonds A, Apondi E, Adedimeji A, et al. Traversing the cascade: urgent research priorities for implementing the \&apos; treat all\&apos; strategy for children and adolescents living with HIV in sub-Saharan Africa. Journal of Virus Eradication. 2018;4(Suppl 2):406.

6. Enane LA, Vreeman RC, Foster C. Retention and adherence: global challenges for the long-term care of adolescents and young adults living with HIV. Current Opinion in HIV and AIDS. 2018;13(3):212-9.

7. Asaolu IO, Gunn JK, Center KE, Koss MP, Iwelunmor JI, Ehiri JE. Predictors of HIV Testing among Youth in Sub-Saharan Africa: A Cross-Sectional Study. PLoS ONE. 2016;11(10):e0164052-12.

8. UNAIDS. Ending AIDS: progress towards the 90-90-90 targets: UNAIDS; 2017 [updated Sep 06. 1-198]. Available from: http://www.unaids.org/en/resources/documents/2017/20170720_Global_AIDS_update_2017.

9. Desmonde S, Tanser F, Vreeman R, Takassi E, Edmonds A, Lumbiganon P, et al. Access to antiretroviral therapy in HIV-infected children aged 0-19 years in the International Epidemiology Databases to Evaluate AIDS (leDEA) Global Cohort Consortium, 2004-2015: A prospective cohort study. PLoS Medicine. 2018;15(5):e1002565-20. 
10. Kariminia A, Law M, Davies M-A, Vinikoor M, Kaloustian KW, Leroy V, et al. Mortality and losses to follow-up among adolescents living with HIV in the leDEA global cohort collaboration. Journal of the International AIDS Society. 2018;21(12):e25215.

11. UNAIDS. UNAIDS Estimates 2018 [Available from: http://aidsinfo.unaids.org/.

12. Ochako R, Mbondo M, Aloo S, Kaimenyi S, Thompson R, Temmerman M, et al. Barriers to modern contraceptive methods uptake among young women in Kenya: a qualitative study. BMC Public Health. 2015;15(1):118.

13. Self A, Chipokosa S, Misomali A, Aung T, Harvey SA, Chimchere M, et al. Youth accessing reproductive health services in Malawi: drivers, barriers, and suggestions from the perspectives of youth and parents. Reprod Health. 2018;15(1):108.

14. WHO. Adolescent pregnancy 2018 [updated Feb 23. Available from: https://www.who.int/newsroom/fact-sheets/detail/adolescent-pregnancy.

15. Kenya CAo. First Quarter Sector Statistics Report for the Communications Authority of Kenya.

16. Namunwa K. Kenya leads Africa in Smartphone usage. Business Today. 2019 March 11, 2019.

17. Lester RT. Ask, don't tell - mobile phones to improve HIV care. New England Journal of Medicine. 2013;369(19):1867-8.

18. Dlamini BR, Mabuza P, Thwala-Tembe M, Masangane Z, Dlamini P, Simelane E. Are adolescents and youth programs missing the real targets? Analysis of socio-cultural factors influencing use of sexual reproductive health services by young people in Swaziland. Journal of AIDS and Clinical Research. 2017;8(4):684.

19. Hamzah L, Hamlyn E. Sexual and reproductive health in HIV-positive adolescents. Current Opinion in HIV and AIDS. 2018;13(3):230-5.

20. Badawy SM, Kuhns LM. Texting and Mobile Phone App Interventions for Improving Adherence to Preventive Behavior in Adolescents: A Systematic Review. JMIR mHealth and uHealth. 2017;5(4):e5017.

21. Horvath T, Azman H, Kennedy GE, Rutherford GW. Mobile phone text messaging for promoting adherence to antiretroviral therapy in patients with HIV infection. Cochrane Database of Systematic Reviews. 2012;376(9755):1838-3.

22. Saberi P, Siedle-Khan R, Sheon N, Lightfoot M. The Use of Mobile Health Applications Among Youth and Young Adults Living with HIV: Focus Group Findings. AIDS Patient Care and STDs. 2016;30(6):254-60.

23. Smillie K, Jane, Borek NV, Kop MLvd, Lukhwaro A, Li N, et al. Mobile health for early retention in HIV care: a qualitative study in Kenya (WelTel Retain). African Journal of AIDS Research. 2015;13(4):3318.

24. Smillie K, Van Borek N, Abaki J, Pick N, Maan EJ, C KFR, et al. A Qualitative Study Investigating the Use of a Mobile Phone Short Message Service Designed to Improve HIV Adherence and Retention in Care in Canada (WelTel BC1). Journal of the Association of Nurses in AIDS Care. 2014;25(6):614-25. 
25. AMPATH. HIV \& AIDS [Available from: https://www.ampathkenya.org/hiv-aids.

26. Vreeman RC, Nyandiko WM, Marete I, Mwangi A, McAteer Cl, Keter A, et al. Evaluating a patientcentred intervention to increase disclosure and promote resilience for children living with HIV in Kenya. AIDS. 2019;33:S93-S101.

27. UNFPA. The Evaluation of Comprehensive Sexuality Education Programmes: A Focus on the Gender and Empowerment Outcomes. New York; 2015.

28. Sidze EM, Stillman M, Keogh S, Mulupi S, Egesa CP, Leong MM, et al. From Paper to Practice: Sexuality Education Policies and Their Implementation in Kenya. New York: Guttmacher Institute; 2017.

29. CIPHER. Global research agendas for paediatric and adolescent HIV Geneva: International AIDS Society, World Health Organization; [Available from: https://www.iasociety.org/HIVProgrammes/Programmes/Paediatrics-CIPHER/Setting-global-research-priorities-for-children-andadolescents-living-with-HIV.

30. Statistics KNBo. Kenya Demographic and Health Survey 2014. Nairobi; 2014.

31. Belzer M. A pilot study using cell phone interactions to improve HIV medication adherence in adolescents who have previously failed antiretroviral therapy. Journal of Adolescent Health. 2013;52(2):S7.

32. Hightow-Weidman LB, Muessig KE, Bauermeister J, Zhang C, LeGrand S. Youth, technology, and HIV: recent advances and future directions. Current HIV/AIDS Reports. 2015;12(4):500-15.

33. Jeffries C, Ross P, Matoff-Stepp S, Thompson R, Harris JL, Uhrig JD, et al. Ucare4life: Mobile texting to improve HIV care continuum outcomes for minority youth. Topics in Antiviral Medicine. 2016;24(E1):427.

34. Liu AY, Vittinghoff E, von Felten P, Amico KR, Anderson PL, Lester R, et al. Randomized Controlled Trial of a Mobile Health Intervention to Promote Retention and Adherence to Pre-exposure Prophylaxis among Young People at Risk for Human Immunodeficiency Virus: The EPIC Study. Clinical infectious diseases : an official publication of the Infectious Diseases Society of America. 2018.

35. Whiteley L, Brown LK, Mena L, Craker L, Arnold T. Enhancing health among youth living with HIV using an iPhone game. AIDS Care. 2019:1-13.

\section{Figures}




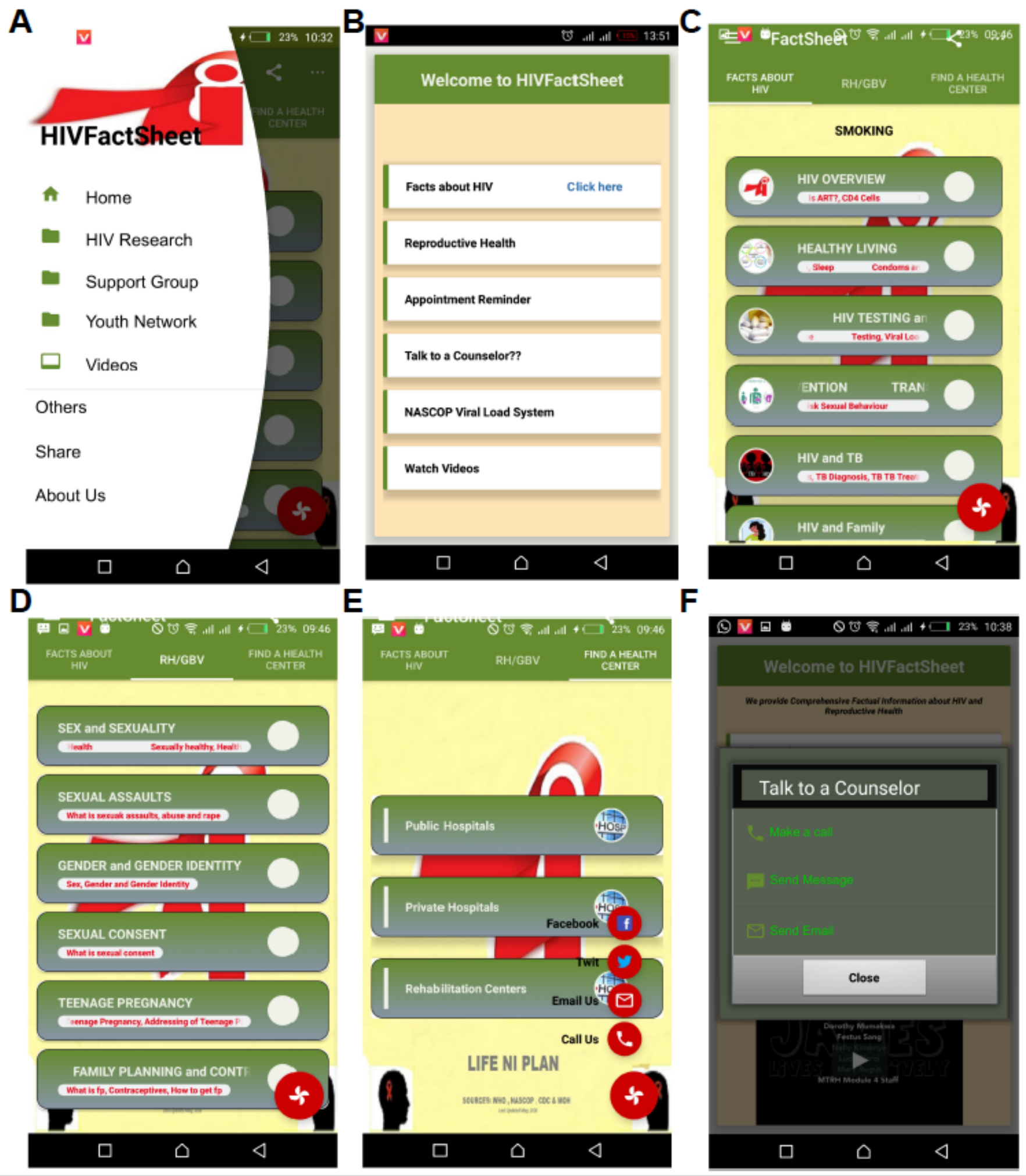

\section{Figure 1}

creenshots from the HIVFactSheet mobile application designed and implemented by youth peer mentors. The HIVFactSheet app incorporates multiple supports for adolescents to access and navigate HIV and sexual and reproductive health care. These include A and B, resources for education on HIV and sexual and reproductive health, appointment reminders; $C$ and $D$, specific education on a number of HIV and sexual and reproductive health topics; E, listings for clinical sites to access HIV and sexual and 
reproductive health care; and F, a portal to text, e-mail, or call a youth peer mentor for individualized support and care referral where needed.

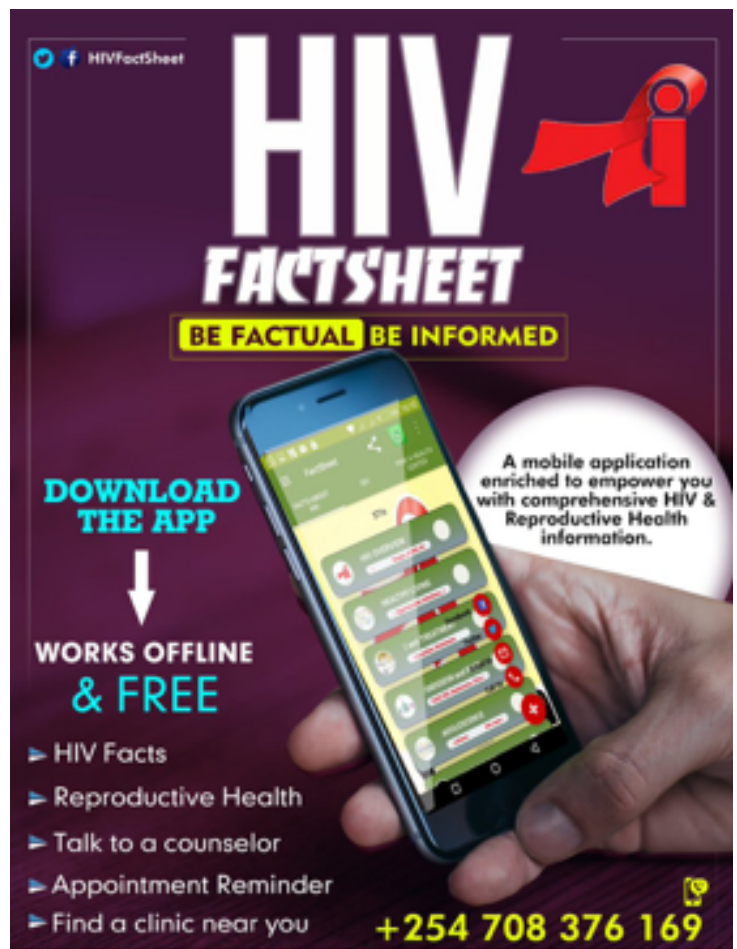

(5) EUSAID tampath

\section{Figure 2}

A promotional poster for HIVFactSheet, a mobile application designed and implemented by youth peer mentors. Promotional posters for the HIVFactSheet app were displayed in the Rafiki Center for Excellence Adolescent Health at Moi Teaching and Referral Hospital in Eldoret, Kenya. The app was promoted on social "fun days" at the clinic and on social media. 